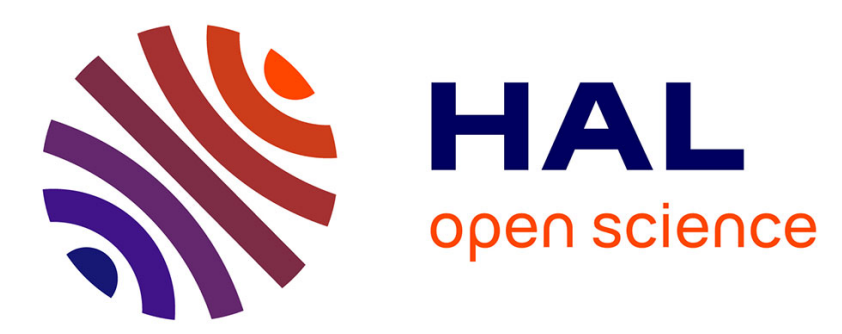

\title{
Blind mobile sensor calibration using an informed nonnegative matrix factorization with a relaxed rendezvous model
}

Clément Dorffer, Matthieu Puigt, Gilles Delmaire, Gilles Roussel

\section{- To cite this version:}

Clément Dorffer, Matthieu Puigt, Gilles Delmaire, Gilles Roussel. Blind mobile sensor calibration using an informed nonnegative matrix factorization with a relaxed rendezvous model. 41st IEEE International Conference on Acoustics, Speech, and Signal Processing (ICASSP 2016), Mar 2016, Shanghai, China. pp.2941-2945, 10.1109/ICASSP.2016.7472216 . hal-01367338

\section{HAL Id: hal-01367338 \\ https://hal.science/hal-01367338}

Submitted on 25 Mar 2018

HAL is a multi-disciplinary open access archive for the deposit and dissemination of scientific research documents, whether they are published or not. The documents may come from teaching and research institutions in France or abroad, or from public or private research centers.
L'archive ouverte pluridisciplinaire HAL, est destinée au dépôt et à la diffusion de documents scientifiques de niveau recherche, publiés ou non, émanant des établissements d'enseignement et de recherche français ou étrangers, des laboratoires publics ou privés. 


\title{
BLIND MOBILE SENSOR CALIBRATION USING AN INFORMED NONNEGATIVE MATRIX FACTORIZATION WITH A RELAXED RENDEZVOUS MODEL
}

\author{
Clément Dorffer, Matthieu Puigt, Gilles Delmaire, and Gilles Roussel \\ Univ. Littoral Côte d'Opale, EA 4491 - LISIC, F-62228 Calais, France
}

\begin{abstract}
In this paper, we consider the problem of blindly calibrating a mobile sensor network-i.e., determining the gain and the offset of each sensor-from heterogeneous observations on a defined spatial area over time. For that purpose, we previously proposed a blind sensor calibration method based on Weighted Informed Nonnegative Matrix Factorization with missing entries. It required a minimum number of rendezvous-i.e., data sensed by different sensors at almost the same time and place-which might be difficult to satisfy in practice. In this paper we relax the rendezvous requirement by using a sparse decomposition of the signal of interest with respect to a known dictionary. The calibration can thus be performed if sensors share some common support in the dictionary, and provides a consistent performance even if no sensors are in exact rendezvous.
\end{abstract}

Index Terms - Blind calibration, mobile sensor networks, informed nonnegative matrix factorization, sparse data analysis

\section{INTRODUCTION}

Crowdsensing is a new way to acquire measurements from a mobile sensor network [1]. This technique exploits the data aggregated by mobile devices - e.g., smartphones - from a crowd of volunteers along their daily moves. The data are then collected and fused to improve their reliability. Currently, crowdsensing appears to be an innovative way to deploy a massive quantity of mobile sensors along a possibly large area and during a long time, with a limited cost. In crowdsensing applications, most sensors-which are usually embedded in mobile devices - are low cost and must be remotely calibrated, as it may not be possible to request these devices to regularly go to a laboratory in order to perform sensor calibration. As a consequence, specific Blind Mobile Sensor Calibration (BMSC) methods ${ }^{1}$ have been proposed in, e.g., [9-12]. All these methods are exploiting the rendezvous model [13] which assumes that sensors in the same spatio-temporal vicinity should acquire the same data. Contrary to [9], the authors in [10-12] consider that some of the available sensors are calibrated. In particular, the methods in $[10,11]$ use a multi-hop calibration structure: calibrated sensors are used to calibrate uncalibrated ones which lie in the same vicinity. These sensors are then considered as calibrated and used to calibrate other sensors when they move. The operation is then repeated until there is no more uncalibrated sensors. Such a multi-hop calibration procedure might suffer from calibration error propagation. On the contrary, in our previous work [12], we revisited BMSC as an informed matrix

\footnotetext{
This work was funded by the "OSCAR" project within the Région Nord Pas de Calais "Chercheurs Citoyens" Program.

${ }^{1}$ It should be noticed that calibration may refer to several different-while sometimes linked-problems and have been tackled, e.g., for fixed sensor gain calibration [2,3], gain/offset calibration [4] or gain/phase calibration
} [5-8].
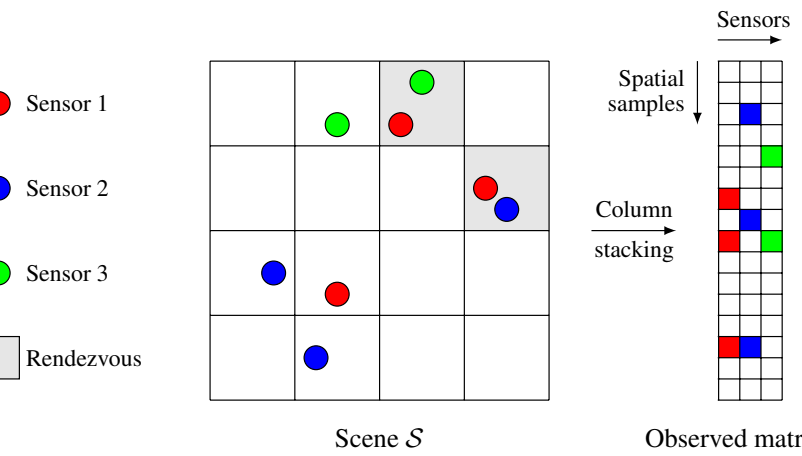

Observed matrix $X$

Fig. 1. From a scene $\mathcal{S}$ (with $n=16$ spatial samples, $m=3$ sensors and 2 rendezvous) to the data matrix $X$ (white pixels mean no observed value).

factorization problem which provided the calibration of all the sensors at the same time. Our approach assumed the sensor network to be dense enough, which might be not satisfied in large areas where few sensors lie.

In this paper, we thus propose a novel calibration method with a relaxed rendezvous model. Indeed, we assume that the sensed physical phenomenon can be sparsely represented in a given dictionary, which is used to regularize the calibration even if the sensors are not in rendezvous.

The remainder of the paper is organized as follows. We introduce the BMSC problem in Section 2 and recall our previous approach in Section 3. We then propose our extended method in Section 4 and investigate its performance in Section 5. Section 6 provides the conclusion and the future work directions of this paper.

\section{PROBLEM STATEMENT}

In this paper, we assume that a geographical area is observed by $m$ heterogeneous, geolocalized, time-stamped, mobile, and possibly uncalibrated sensors along time. We now introduce the first definitions and assumptions used in this paper.

Definition 1 ( [13]) A rendezvous is a temporal and spatial vicinity between two sensors.

The vicinity is characterized by $\Delta_{t}$ and $\Delta_{d}$ which respectively correspond to a time duration and a distance. Depending on the sensed physical phenomenon, the values of $\Delta_{t}$ and $\Delta_{d}$ may drastically vary [13].

Definition 2 ( [12]) A scene $\mathcal{S}$ is a discretized area observed during a time interval $\left[t, t+\Delta_{t}\right)$. The size of the spatial pixels are set so that any couple of points inside the same pixel have a distance below $\Delta_{d}$ 
A scene can be seen as a grid of locations where sensors go to and where they sense a physical phenomenon. When two sensors share a common position in a scene, they are in rendezvous (see Fig. 1).

Assuming an affine calibration model-i.e., that each of the $m$ sensors provides an output linked to the sensor phenomenon via an affine relationship — an available data point $x_{i j}$ provided by Sensor $j$ and associated to the $i^{\text {th }}$ location of $\mathcal{S}$ then reads

$$
x_{i j} \simeq y_{i} \cdot \alpha_{j}+\beta_{j},
$$

where $\alpha_{j}$ and $\beta_{j}$ are the unknown gain and offset associated with Sensor $j$, respectively, and $y_{i}$ is the sensed phenomenon at Location $i$. By respectively defining $G$ and $F$ the $n \times 2$ and $2 \times m$ matrices

$$
G \triangleq\left[\begin{array}{ccc}
y_{1} & \cdots & y_{n} \\
1 & \cdots & 1
\end{array}\right]^{T} \text { and } F \triangleq\left[\begin{array}{ccc}
\alpha_{1} & \cdots & \alpha_{m} \\
\beta_{1} & \cdots & \beta_{m}
\end{array}\right],
$$

the matrix form of Eq. (1)—when all the data points $x_{i j}$ are available - can then be expressed as a low-rank matrix which reads

$$
X \simeq G \cdot F .
$$

This factorization can be performed in practice if $X$ is wellconditioned, which implies that we observe enough diversity in the first column of $G$ and in the rows of $F$.

We also assume that one sensor-say Sensor $m$-is calibrated ${ }^{2}$ and that its calibration parameters are respectively equal to ${ }^{3}$

$$
\alpha_{m}=1 \quad \text { and } \quad \beta_{m}=0 .
$$

i.e., if the value $x_{i m}$ is available, we get $x_{i m} \simeq y_{i}$. Lastly, we assume that the observed phenomenon and the sensor calibration parameters are nonnegative, which implies that the problem (3) can be tackled by Weighted Informed Nonnegative Matrix Factorisation (WINMF), as we show in the next section.

\section{PREVIOUSLY PROPOSED BMSC METHOD}

As explained above, we aim to factorize $X$ as a product $G \cdot F$ of nonnegative matrices $G$ and $F$. This turns out to be a WINMF problem which can be tackled by, e.g., the approach in [14] —extending the multiplicative update NMF approaches in [15-17] - that we used and generalized in [12]. Indeed, the last columns in $G$ and $F$ are perfectly known as well as some entries in the first column of $G$. This information can be used as a specific parameterization where only the free parts of $G$ and $F$ are updated [12,14]. WINMF then reads

$$
\begin{gathered}
\{G, F\}=\quad \arg \min _{\tilde{G} \geq 0, \tilde{F} \geq 0}\|W \circ(X-\tilde{G} \cdot \tilde{F})\|_{\mathcal{F}}^{2}, \\
\text { s.t. } \quad \tilde{G}=\Omega_{G} \circ \Phi_{G}+\bar{\Omega}_{G} \circ \Delta_{G}, \\
\tilde{F}=\Omega_{F} \circ \Phi_{F}+\bar{\Omega}_{F} \circ \Delta_{F},
\end{gathered}
$$

where $W$ is the $n \times m$ weight matrix whose entries are defined as

$$
w_{i j} \triangleq \begin{cases}0 & \text { if } x_{i j} \text { is not available } \\ \rho_{j} & \text { otherwise }\end{cases}
$$

\footnotetext{
${ }^{2}$ If no calibrated sensor is available, it is still possible to perform a relative calibration, thus providing some consistency in the sensor responses [4].

${ }^{3}$ In the considered crowdsensing application, some highly accurate and calibrated sensors provide measurements of the sensed phenomenon. As we are not provided neither their calibration model nor their calibration parameters, we fix $\alpha_{m}$ and $\beta_{m}$ as in Eq. (4) so that the observed values are equal to the calibrated measurements.
}

where $\rho_{j}$ is a weight coefficient associated with Sensor $j$, o denotes the Hadamard product, $\Omega_{G}$ (respectively $\Omega_{F}$ ) is a binary matrix informing the presence/absence of constraints in $G$ (respectively $F$ ), $\Phi_{G}$ (respectively $\Phi_{F}$ ) is the matrix containing the set entries in $G$ (respectively $F$ ),$\Delta_{G}$ (respectively $\Delta_{F}$ ) is the matrix of the free parameters in $G$ (respectively in $F$ ), and $\bar{\Omega}_{G} \triangleq \mathbb{1}_{n \times 2}-\Omega_{G}$ (respectively $\bar{\Omega}_{F} \triangleq \mathbb{1}_{2 \times m}-\Omega_{F}$ ), where $\mathbb{1}_{i \times j}$ is the $i \times j$ matrix of ones.

The solution of Eq. (5) can be estimated through an alternating strategy which aims to solve both optimization sub-problems:

$$
\begin{gathered}
F=\quad \arg \min _{\tilde{F} \geq 0}\|W \circ(X-G \cdot \tilde{F})\|_{\mathcal{F}}^{2}, \\
\text { s.t. } \quad \tilde{F}=\Omega_{F} \circ \Phi_{F}+\bar{\Omega}_{F} \circ \Delta_{F},
\end{gathered}
$$

and

$$
\begin{gathered}
G=\quad \arg \min _{\tilde{G} \geq 0}\|W \circ(X-\tilde{G} \cdot F)\|_{\mathcal{F}}^{2}, \\
\text { s.t. } \quad \tilde{G}=\Omega_{G} \circ \Phi_{G}+\bar{\Omega}_{G} \circ \Delta_{G} .
\end{gathered}
$$

In $[12,14]$, we proposed the following update rules to solve these subproblems:

$$
F \leftarrow \Phi_{F}+\Delta_{F} \circ \bar{\Omega}_{F} \circ\left[\frac{G^{T}\left(W \circ\left(X-G \cdot \Phi_{F}\right)^{+}\right)}{G^{T}\left(W \circ\left(G \cdot \Delta_{F}\right)\right.}\right],
$$

and

$$
G \leftarrow \Phi_{G}+\Delta_{G} \circ \bar{\Omega}_{G} \circ\left[\frac{\left(W \circ\left(X-\Phi_{G} \cdot F\right)^{+}\right) F^{T}}{\left(W \circ\left(\Delta_{G} \cdot F\right)\right) F^{T}}\right],
$$

where the superscript ${ }^{+}$denotes the function defined as $(z)^{+} \triangleq$ $\max \{\epsilon, z\}$, where $\epsilon$ is a small user-defined threshold.

As initializing the NMF is known to be tricky, we proposed to use a matrix completion algorithm to complete $X$ and then, to replace the missing entries in the first column of $G$ by the corresponding entries in the last column of $X$. Finally, $F$ was initialized from the completed matrices $X$ and $G$ using nonnegative least squares.

In [12], we showed that if the number of measurements is large enough and if there are enough rendezvous between sensors, the WINMF-based calibration is accurate. However, in a practical crowdsensing application, the above assumption on the number of measurements and rendezvous is more likely to be unsatisfied if a few sensors observe a large area. This implies a poor calibration accuracy, as shown in, e.g., [12]. We thus propose in the next section an extension of this method which uses a relaxed rendezvous assumption.

\section{BMSC WITH RELAXED RENDEZVOUS}

In order to improve the robustness of the method against the number of rendezvous, we make one more assumption on the sensed phenomenon, leading to an extra constraint in the optimization problem (5). We assume that the sensed phenomenon $y$ on a scene $\mathcal{S}$ allows a sparse decomposition according to a known $\overline{\text { dictionary }} \mathcal{D}$, i.e.,

$$
\exists \underline{a} \in \mathbb{R}^{q} \text { s.t. } \underline{y} \simeq \mathcal{D} \cdot \underline{a},
$$

with $p \ll q$ non-zero elements in $\underline{a}$. From Eq. (2), the first row in $G$-denoted $\underline{g}_{1}$ below-is equal to $\underline{y}$ and $G$ may be rewritten as

$$
G=\left[\begin{array}{ccc} 
& \underline{a}^{T} \cdot \mathcal{D}^{T} & \\
1 & \cdots & 1
\end{array}\right]^{T}
$$


Injecting this new structure of $G$ in the relationship (3) then leads to the modified optimization problem:

$$
\begin{aligned}
&\{G, F, \underline{a}\}= \arg \min _{\tilde{G} \geq 0, \tilde{F} \geq 0, \underline{\tilde{a}}}\|W \circ(X-\tilde{G} \cdot \tilde{F})\|_{\mathcal{F}}^{2} \\
&+\lambda \cdot\left\|\underline{\tilde{g}}_{1}-\mathcal{D} \cdot \underline{\tilde{a}}\right\|_{2}^{2}+\mu\|\underline{\tilde{a}}\|_{1} \\
& \text { s.t. } \quad \tilde{G}=\Omega_{G} \circ \Phi_{G}+\bar{\Omega}_{G} \circ \Delta_{G}, \\
& \tilde{F}=\Omega_{F} \circ \Phi_{F}+\bar{\Omega}_{F} \circ \Delta_{F},
\end{aligned}
$$

where $\lambda$ and $\mu$ are weights associated with the penalization terms in Eq. (13) and $\tilde{g}_{1}$ stands for the first column of $\tilde{G}$. It should be noticed that such an optimization problem differs from classical NMF with sparse assumptions [18] since a dictionary is added into the decomposition. Moreover, its resolution is tricky since such a problem is non-convex with respect to the three unknowns $G, F$, and $\underline{a}$ but it can be decomposed into three convex sub-problems where we aim to successively and alternatingly estimate one unknown. In that case, $F$ can be estimated by solving Eq. (7), $G$ using

$$
\begin{gathered}
G=\arg \min _{\tilde{G} \geq 0}\|W \circ(X-\tilde{G} \cdot F)\|_{\mathcal{F}}^{2}+\lambda \cdot\left\|\underline{\tilde{g}}_{1}-\mathcal{D} \cdot \underline{a}\right\|_{2}^{2}, \\
\text { s.t. } \quad \tilde{G}=\Omega_{G} \circ \Phi_{G}+\bar{\Omega}_{G} \circ \Delta_{G},
\end{gathered}
$$

and $\underline{a}$ while solving

$$
\underline{a}=\arg \min _{\underline{\tilde{a}}}\left\|\underline{\tilde{g}}_{1}-\mathcal{D} \cdot \underline{\tilde{a}}\right\|_{2}^{2}+\mu\|\underline{\tilde{a}}\|_{1} .
$$

We then solve these problems alternately for each unknown. In order to derive the update rules for $G$, we take into account the penalization term in Eq. (14) by considering a new NMF problem where we add a new column in $W, X$, and $F$, respectively, thus providing new matrices defined as follows:

$$
\mathcal{W} \triangleq\left[W, \sqrt{\lambda} \cdot \mathbb{1}_{n \times 1}\right], \mathcal{X} \triangleq[X, \mathcal{D} \cdot a], \text { and } \mathcal{F} \triangleq\left[F,\left(\begin{array}{l}
1 \\
0
\end{array}\right)\right]
$$

Provided an accurate sparse approximation of $g_{1}$, all the sensors are in rendezvous - since the last column of $\mathcal{X}$ is fully known-which highly relax the original assumption and which was never considered in the literature to the best of the authors' knowledge.

Using these matrices, it is then straightforward to see that the problems (14) and (8) are equivalent-except that we respectively replace $W, X$, and $F$ by $\mathcal{W}, \mathcal{X}$, and $\mathcal{F}$ in Eq. (8) — so that we can use the update rules (10). $F$ can be estimated using the update rules (9). Lastly, the optimization problem in Eq. (15) classically provides sparse approximations of $\hat{g}_{1}$. Such a problem can be solved, e.g., with Basis Pursuit Denoising or its numerous extensions [19], for which it is well known that the value of $\mu$ should be carefully chosen with respect to the additive noise variance. Altenatively, greedy approaches were proposed and in this paper, we used Orthogonal Matching Pursuit (OMP) [20]—which iteratively incorporates atoms into the sparse decomposition-as we found it to provide accurate approximations in preliminary tests. The algorithm of our proposed WINMF method is provided in Algorithm 1.

Let us stress again the advantages of the proposed formulation of WINMF: $\underline{a}$ constraints $\underline{g}_{1}$ to be a linear combination of a few atoms of the dictionary. This allows the method not to require anymore exact rendezvous but relaxed ones. However, we still need at least two measurements per sensor in order to be able to perform the calibration. Moreover, we notice in experiments that the convergence of this improved WINMF calibration is greatly accelerated, which was one of the limitations of the method in [12]. We provide in the next section a detailed performance analysis of the approach proposed in this paper.

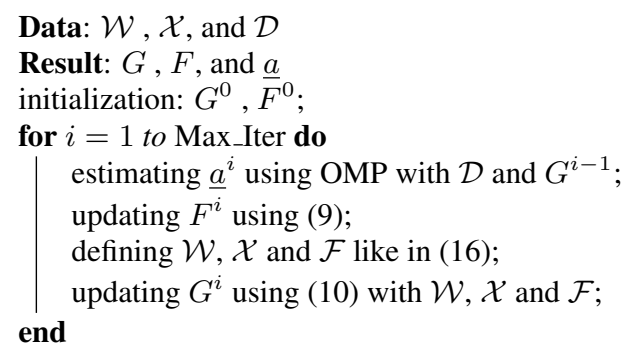

Algorithm 1: Penalized NMF algorithm.

\section{SIMULATIONS}

In this section, we aim to investigate the enhancement provided by our proposed informed NMF method for BMSC. For that purpose, we consider the simulation of crowdsensing-like particulate matter sensing used in [12]. The scene is a $10 \times 10$ discretized area (the length of $\underline{y}$ is thus equal to $n=100$ ) which is observed by $m=$ 26 sensors, i.e., $m-1$ uncalibrated and mobile dust sensors [21] connected to mobile devices and one calibrated, high quality, and mobile sensor ${ }^{4}$.

The observed concentrations in $\underline{y}$ range between 0 and $0.5 \mathrm{mg} / \mathrm{m}^{3}$, for which the sensor response is assumed to be affine [21]. For each uncalibrated sensor, each observed data point represents a nonnegative voltage linked to the corresponding ground truth point in $\underline{y}$ according to Eq. (1). We then get a $100 \times 26$ theoretical observation matrix for which we randomly keep $k+l$ samples in $X$ only, where $k$ (respectively, $l$ ) is the number of calibrated (respectively, uncalibrated) sensor samples-with $k \ll l$-hence providing the irregular spatial sampling over the scene. Lastly, Gaussian noise realizations may be added to the observed uncalibrated sensor data and the weight coefficients $\rho_{j}$ defined in Eq. (6) are set to $\rho_{j}=1$. The dictionary is constructed with $q=62$ different atoms. Actually, it was designed so that $\underline{g}_{1}$ could be reconstructed from $p=2$ different atoms. As in [12], we aim to explore the influence of the number of rendezvous between calibrated and uncalibrated sensors, the number of missing entries in $X$ and the influence of the input SNR to the BMSC performance. For each test condition-i.e., one number of rendezvous, one proportion of missing entries, or one input SNR -25 simulations are performed. In each run, we randomly set the positions of the samples in $X$ in the three experiments and we generate different noise realizations in the last one. The number $k$ of calibrated sensor values in the $m$-th column of $X$ is set to $k=4$ in all the tests.

Except when we make these values vary, the proportion of uncalibrated sensors to have rendezvous with calibrated ones, and the proportion of missing entries in $X$ are set to $30 \%$ and $90 \%$, respectively. In addition to the noiseless case, the input SNR varies from 10 to $90 \mathrm{~dB}$. Lastly, the methods are stopped after 200000 iterations. We also vary the value of $\lambda$ from 0.1 to 10 but do not notice any major influence of this parameter. The plots provided in this paper are obtained with $\lambda=10$.

\footnotetext{
${ }^{4}$ Actually, we get $k$ fixed, calibrated, and accurate sensors whose obtained values are modeled as those of the $m$-th sensor in the BMSC problem.
} 


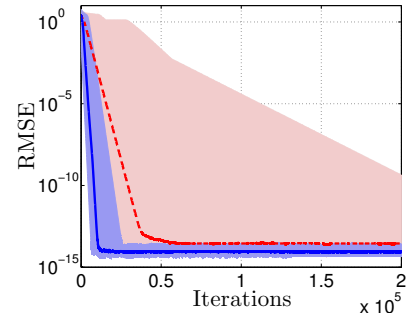

(a)

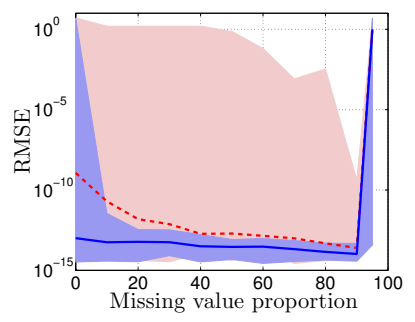

(b)

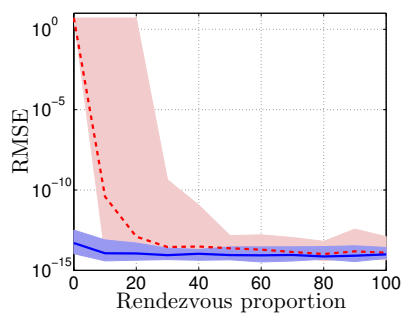

(c)

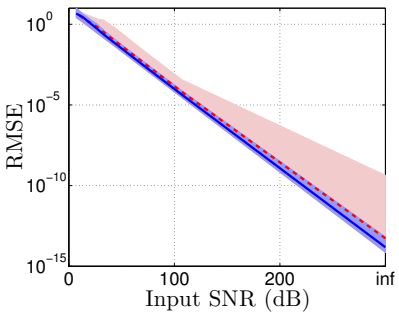

(d)

Fig. 2. Evolution of the RMSE according to (a) the number of iterations, (b) the missing value proportion, (c) the rendezvous proportion, and (d) the input SNR .

The calibration error is quantified by the Root Mean Square Error (RMSE) which is computed between the vector of the true unknown $^{5}$ gains $\underline{\alpha} \triangleq\left[\alpha_{1} \cdots \alpha_{m-1}\right]$ and the vector of the reconstructed ones $\underline{\hat{\alpha}}=\left[\hat{\alpha}_{1} \cdots \hat{\alpha}_{m-1}\right]$, i.e.,

$$
\operatorname{RMSE}(\underline{\alpha}, \underline{\hat{\alpha}})=\frac{\|\underline{\alpha}-\underline{\hat{\alpha}}\|_{2}}{\sqrt{m-1}} .
$$

Similarly, the RMSE between theoretical and estimated offsets is computed but is not shown in this paper for space considerations.

Figure 2 shows the different plots of the different experiments realized in this paper. The red dashed line (respectively the blue line) corresponds to the median RMSE obtained with our previous approach (respectively the proposed method) applied on 25 independent tests while the red (respectively the blue) colored area corresponds to the envelope of the RMSEs obtained with our previous method (respectively the proposed approach).

Figure 2.(a) shows the RMSE evolution with respect to the iterations. One can see that the slowest RMSE convergence curve of our proposed BMSC approach is much faster than the median RMSE convergence curve provided by our previous method. The median RMSE of our proposed approach is almost constant after 20000 iterations - when the median RMSE of our previous one needs 60000 iterations-while being lower than the median RMSE of our previous method.

Figure 2.(b) shows the influence of the missing value proportion on the calibration performance. Here again, our new approach outperforms the previous one. It should be noticed that there is one high RMSE with no missing entry in $X$ in one of the tests, hence a large envelope. Actually, in this particular case, the WINMF did not converge, hence a large RMSE which should be lower if we ran more iterations. When $95 \%$ of the data are missing, one of the assumptions-i.e., the diversity of the measurements or their minimal number per sensors-is not satisfied, hence a large median RMSE. However, the lowest part of the blue envelope shows a RMSE around 1e-14, which is not reached without the dictionary, hence the interest of our proposed approach.

We plot in Fig. 2.(c) the influence of the number of rendezvous between calibrated and uncalibrated sensors when $90 \%$ of the data are missing. As expected, the calibration performance reached by our proposed calibration technique takes advantage of the dictionary and provides a consistent performance, even when no rendezvous is met. On the contrary, our previous approach provides much higher RMSEs when the rendezvous proportion is below $20 \%$.

\footnotetext{
${ }^{5}$ Due to Eq. (4), $\alpha_{m}$ is perfectly known, thus resulting in $m-1$ unknown gains to be estimated.
}

Lastly, Fig. 2.(d) shows the influence of the input SNR. As expected, the RMSE increases when the input SNR decreases. Both approaches provide similar performance in the noisy cases, which was expected as we do not use the dictionary to denoise the observed data.

\section{CONCLUSION}

In this paper, we revisited blind mobile sensor calibration as a matrix factorization problem. Assuming any of the matrices in the factorization to be nonnegative and the sensed phenomenon to be sparsely represented in a dictionary, we improved our previous informed NMF [12] for the considered application. The approach was shown to be robust to the number of missing entries and to the number of rendezvous between calibrated and uncalibrated sensors. However, some assumptions-e.g., the minimum number of measurements per sensor or the fact that $X$ is well-conditioned-might seem restrictive. It should be noticed that the approach proposed in this paper can be extended to the case of multiple scenes, by stacking all the observed — and sufficiently different - matrices in one unique well-conditioned matrix, so that we multiply the number of measurements. In that case, it is more likely that the calibration assumptions will be satisfied.

In future work, we aim to explore several directions. As mentioned above, joint-factorization will be investigated. Moreover, the NMF method proposed in this paper is an extension of the Lee and Seung multiplicative update algorithm, which is known to be slow to converge when the size of the data matrix is large. Extending recent and fast NMF methods to our informed framework will be considered. Lastly, we will investigate other calibration models, wellsuited to gaz sensors for example.

\section{REFERENCES}

[1] R.K. Ganti, F. Ye, and H. Lei, "Mobile crowdsensing: current state and future challenges," IEEE Communications Magazine, vol. 49, no. 11, pp. 32-39, November 2011.

[2] H. Carfantan and J. Idier, "Statistical linear destriping of satellite-based pushbroom-type images," IEEE Trans. on Geoscience and Remote Sensing, vol. 48, no. 4, pp. 1860-1871, April 2010.

[3] C. Schulke, F. Caltagirone, F. Krzakala, and L. Zdeborova, "Blind calibration in compressed sensing using message passing algorithms," in NIPS 26, 2013, pp. 566-574. 
[4] L. Balzano and R. Nowak, "Blind calibration of sensor networks," in Proc. of IPSN, 2007, pp. 79-88.

[5] P.H. Schönemann, "On metric multidimensional unfolding," Psychometrika, vol. 35, no. 1, pp. 349-366, March 1970.

[6] M. Pollefeys and D. Nister, "Direct computation of sound and microphone locations from time-difference-of-arrival data," in Proc. of ICASSP, March 2008, pp. 2445-2448.

[7] S. Ando and N. Ono, "A Bayesian theory of cooperative calibration and synchronization in sensor networks," Trans. of SICE, vol. E-S-1, pp. 21-26, 2005.

[8] C. Bilen, G. Puy, R. Gribonval, and L. Daudet, "Convex optimization approaches for blind sensor calibration using sparsity," IEEE Trans. on Signal Processing, vol. 62, no. 18, pp. 4847-4856, Sept 2014.

[9] B.-T. Lee, S.-C. Son, and K. Kang, "A blind calibration scheme exploiting mutual calibration relationships for a dense mobile sensor network," IEEE Sensors Journal, vol. 14, no. 5, pp. 1518-1526, May 2014.

[10] E. Miluzzo, N.D. Lane, A.T. Campbell, and R. Olfati-Saber, "CaliBree: A self-calibration system for mobile sensor networks," in Proc. of DCOSS, 2008, vol. 5067 of LNCS, pp. 314-331.

[11] O. Saukh, D. Hasenfratz, and L. Thiele, "Reducing multi-hop calibration errors in large-scale mobile sensor networks," in Proc. of IPSN, 2015.

[12] C. Dorffer, M. Puigt, G. Delmaire, and G. Roussel, "Blind calibration of mobile sensors using informed nonnegative matrix factorization," in Proc. of LVA-ICA, vol. 9237 of LNCS, pp. 497-505. 2015.

[13] O. Saukh, D. Hasenfratz, C. Walser, and L. Thiele, "On rendezvous in mobile sensing networks," in Proc. of REALWSN, 2014, vol. 281 of $L N C S$, pp. 29-42.

[14] A. Limem, G. Delmaire, M. Puigt, G. Roussel, and D. Courcot, "Non-negative matrix factorization under equality constraints - a study of industrial source identification," Applied Numerical Mathematics, vol. 85, pp. 1-15, November 2014.

[15] D.D. Lee and H.S. Seung, "Learning the parts of objects by non negative matrix factorization," Nature, vol. 401, no. 6755, pp. 788-791, 1999.

[16] D. Guillamet, J. Vitria, and B. Schiele, "Introducing a weighted non-negative matrix factorization for image classification," Pattern Recognition Letters, vol. 24, no. 14, pp. 2447-2454, 2003.

[17] N.-D. Ho, Non negative matrix factorization algorithms and applications, Phd thesis, Université Catholique de Louvain, 2008.

[18] P.O. Hoyer, "Non-negative matrix factorization with sparseness constraint," Journal of Machine Learning Research, vol. 5, pp. 1457-1469, November 2004.

[19] M. Elad, Sparse and Redundant Representations: From Theory to Applications in Signal and Image Processing, Springer, 2010.

[20] Y. Pati, R. Rezaiifar, and P. Krishnaprasad, "Orthogonal Matching Pursuit: recursive function approximation with application to wavelet decomposition," in Proc. of Asilomar Conf. on Signals, Systems and Comput., 1993.
[21] Sharp Corp., "GP2Y1010AU0F compact optical dust sensor," 2006, Datasheet. 